\title{
A model for traffic behaviour evaluation based on a discrete event simulation approach
}

\author{
A. Di Febbraro \& N. Sacco \\ Department of Machines, Energy Systems, and Transportation, \\ University of Genoa, Italy
}

\begin{abstract}
There are various reasons why both freight and passenger transport demands keep growing significantly not only in Europe, but also worldwide. In this framework, the interaction between such demands in urban areas assumes a relevant role in the overall performances of an urban transportation system. In this paper, the special case of a seaport located in an urban area is considered, since such a situation often occurs in cities grown along the coast around an "old" port. Then, to cope with the problem of simply modelling the freight and passenger flows in urban areas, in this paper a coloured Petri net modelling approach is proposed.

Keywords: urban traffic networks, coloured Petri nets, freight and passenger transportation demand.
\end{abstract}

\section{Introduction}

It is well-known that, for different reasons, freight transport keeps growing significantly not only in Europe, but also worldwide. Unfortunately, all the same the share taken by road transport is enlarging, resulting in increasing congestion, environmental harm, and any other kind of costs. In this framework, the interaction between the freight and the passenger transport demand in urban areas assumes a relevant role in the overall performances of an urban transportation system. In this paper, the special case of a seaport located in an urban area is considered. Such a situation, often occurring in cities grown along the coast around an "old" port, is very difficult to cope with from a transportation point of view. In fact, the overall management of the interactions among the different transport modes can become a formidable task. With the aim of evaluating such interactions, it is worth underlining that intermodal freight terminals are typically complex large systems managing 
a huge amount of different goods, often packed in containers. Such a characteristic usually makes the relevant performance evaluation an interesting modelling problem. Then, to cope with this challenge, in this paper, a discrete event based model is proposed. In doing so, intermodal terminals and the whole urban area are considered to be Discrete Event Systems (DES), and the proposed model is implemented by means of Coloured Stochastic Timed Petri Nets (CSTPNs), which can take into account some structural characteristics and constraints of the systems, such as finite capacity and resources, or uncertainty in some parameters of the considered system (see for instance [1]).

The paper is organised as follows. In Section 2 the basic definitions about ordinary and coloured Petri nets are given. In the following Section 3, the considered model of urban traffic network and its main elements are introduced, both from a descriptive and CSTPN points of view. Then Section 4, and Section 5 are devoted, respectively, to a description of the selected case study, and the relevant conclusions.

\section{Basics on Petri Nets}

In this section, the Petri net modelling formalism is briefly described.

\subsection{Stochastic timed Petri nets}

A Stochastic Timed Petri Net (STPN) is a bipartite oriented graph (see [2] for more details) described by the 5-tuple

$$
\mathrm{STPN}=(P, T, \text { Pre, Post }, \Theta)
$$

where $P$ is a set of $n$ places and $T$ is a set of $m$ transitions. The sets $T$ and $P$ are disjoint, that is, $P \cap T=\emptyset$. Then, Pre $: P \times T \rightarrow \mathbb{N}_{0}$ (resp., Post : $P \times T \rightarrow \mathbb{N}_{0}$ ), being $\mathbb{N}_{0}$ the set of the integer number with the null element 0 , is the pre-incidence (resp., post-incidence), matrix whose generic element $\mathbf{P r e}_{i, j}$ (resp., Post $_{i, j}$ ) indicates the weight of the arc exiting from place $p_{i}$ towards its output transition $t_{j}$ (resp., exiting from transition $t_{j}$ towards its output place $p_{i}$ ). Finally, $\Theta=\left\{\Theta_{j}: t_{j} \in T\right\}$ is the clock. The elements $\Theta_{i}$ of the set $\Theta$ may be vectors or scalars. In the first case $\Theta_{j}=\left\{F D_{j}(1), F D_{j}(2), \ldots\right\}$, and its generic element $F D_{j}(h) \in \mathbb{R}_{0}^{+}, h \in \mathbb{N}$, specifies the deterministic delay occurring between the $h^{\text {th }}$ enabling and the firing of transition $t_{j}$. On the other hand, if $\Theta_{j}$ is a scalar, then the real firing delay $F D_{i}(h)$ between the $h^{\text {th }}$ enabling and the $h^{\text {th }}$ firing of transition $t_{j}$ is an extraction of an exponential stochastic variable with mean $\mu=\frac{1}{\Theta_{j}}$. Finally, if $\Theta_{j}=0$, then $t_{j}$ is said "immediate transition".

In addition, for each transition $t_{j},{ }^{\bullet} t_{j}$ and $t_{j}^{\bullet}$ denote the sets of its input and output places. Analogously, for each place $p_{i},{ }^{\bullet} p_{i}$ and $p_{i}^{\bullet}$ denote the sets of its input and output transitions. 
From a graphical point of view, places are represented by means of circles, deterministic transitions are represented by means of black boxes, and stochastic transitions are represented by means of white boxes. Finally, arcs are represented by means of arrows.

For what concerns the state of the system represented by means of a STPN, it is given by the marking of the net, which is an ordered vector in $\mathbb{N}^{n}$, whose generic element $\mathcal{M}_{i}$ gives the number of tokens in place $p_{i}$. From the dynamic point of view, a transition $t_{j}$ is enabled by the $k^{\text {th }}$ marking $\mathcal{M}(k)$ reached by the net, if and only if $\mathcal{M}_{i}(k) \geq \mathbf{P r e}_{i, j}, \forall i \in{ }^{\bullet} t_{j}$. When enabled, transition $t_{j}$ fires for the $k^{\text {th }}$ time after the delay $F D_{j}(k), \mathbf{P r e}_{i, j}$ tokens are removed by each place $p_{i} \in{ }^{\bullet} t_{j}$, and Post $_{h, j}$ tokens are added by each place $p_{h} \in t_{j}^{\bullet}$.

\subsection{Coloured stochastic timed Petri nets}

In this section, the Coloured Stochastic Petri Net (CSTPN) formalism, which has been firstly introduced by Jensen [3], is described. Such a modelling approach is here chosen for its capability of modelling the different kinds of vehicles in an urban traffic network.

Formally, a CSTPN is defined as a bipartite directed graph represented by a 6-tuple

$$
\operatorname{CSTPN}=(P, T, C o, \text { Pre, } \text { Post }, \Theta),
$$

where $P$ is a set of $n$ places and $T$ is a set of $m$ transitions. As in the ordinary Petri nets, the sets $P$ and $T$ are disjoint. Then, $C o$ is the colour function that associates each element in $P \cup T$ with a non empty ordered set of colours in the set of all the possible colours $\mathrm{Cl}$. More precisely, $\mathrm{Co}$ is the function which maps each place $p_{i} \in P$ (resp., transition $t_{j} \in T$ ) to the set of possible token colours $C o\left(p_{i}\right)=\left\{a_{1}, a_{2}, \ldots, a_{i, A_{i}}\right\}$ (resp., $C o\left(t_{j}\right)=\left\{b_{1}, b_{2}, \ldots, b_{j, B_{j}}\right\}$ ) being $A_{i}$ (resp., $B_{j}$ ) the number of possible colours of tokens in $p_{i}$ (resp., $t_{j}$ ). Then, the elements Pre and Post are the pre-incidence and the post-incidence matrices, respectively. In coloured Petri nets, each element $\mathbf{P r e}_{i, j}$ of Pre is itself a matrix of the space $\mathbb{N}_{0}^{A_{i}, B_{j}}$, whose generic element $\operatorname{Pre}_{i, j}(h, k)$ is equal to the weight of the arc from place $p_{i}$, with respect to colour $a_{i, h}$, to transition $t_{j}$, with respect to colour $b_{j, k}$. Analogously, each element of Post $\mathbf{P}_{i, j}$ is a matrix of the space $\mathbb{N}_{0}^{A_{i}, B_{j}}$, whose generic element Pre $_{i, j}(h, k)$ is equal to the weight of the arc from transition $t_{j}$, with respect to colour $b_{j, k}$, to place $p_{i}$ with respect to colour $a_{i, h}$.

Finally, $\Theta$ denotes the timing vector, as in ordinary Petri nets. In this framework, each element $\Theta_{j}$ of $\Theta$, no matter whether it is a constant or a vector, as above explained, may be split into $B_{j}$ terms, each one corresponding to a possible colour of transition $t_{j}$.

For what concerns the state of the system, also in CSTPNs it is represented by the marking of the net, which associates with all the possible token colours in $p_{i}$ a non-negative integer number. Note that, for each place $p_{i}$, the relevant $\mathcal{M}_{i}$ is a vector in $\mathbb{N}^{A_{i}}$, whose $h^{\text {th }}$ component $\mathcal{M}_{i, h}$ is equal to the number of tokens of colour $a_{i, h}$ that are gathered in $p_{i}$. Then, the marking of the whole net is a 
column vector $\mathcal{M}=\left[\mathcal{M}_{1}, \ldots, \mathcal{M}_{n}\right]^{\mathrm{T}}$ gathering the markings of each place $\mathcal{M}_{i}$, $i=1, \ldots, n$.

As regards the dynamics of the net, in the CSTPN any transition $t_{j} \in T$ should be enabled, at the $k^{\text {th }}$ marking $\mathcal{M}(k)$, with respect to the $l^{\text {th }}$ colour of $C o\left(t_{j}\right)$ if and only if the relation $\mathcal{M}_{i, h}(k) \geq \operatorname{Pre}_{i, j}(h, l), \forall h=1, \ldots, A_{i}, \forall p_{i} \in{ }^{\bullet} t_{j}$, is fulfilled.

When an enabled transition $t_{j}$ fires for the $k^{\text {th }}$ time, with respect to the colour $b_{j, l}$, the CSTPN reaches the marking $\mathcal{M}(k+1)$ such that

$$
M_{i}(k+1)=M_{i}(k)+\left[\operatorname{Post}_{i, j}(:, l)-\operatorname{Pre}_{i, j}(:, l)\right]^{\mathrm{T}}, \quad \forall p_{i} \in P,
$$

where the notation $\operatorname{Pre}_{i, j}(:, l)$ (resp., $\left.\operatorname{Post}_{i, j}(:, l)\right)$ indicates the whole $l^{\text {th }}$ column of the matrix Pre $_{i, j}$ (resp., Post Pr,j $_{i}$ ).

In the following, all the places of the considered CSTPN are supposed to have a finite capacity $C_{i}$, which is defined as the "equivalent marking" it can contain at a time. In this framework, the equivalent marking $\mathcal{M}_{i}^{e q}=\left[\alpha_{1}, \ldots, \alpha_{A_{i}}\right] \cdot \mathcal{M}_{i}^{\mathrm{T}}$ is defined as a weighted sum of the elements of $\mathcal{M}_{i}$, being the weights $\alpha_{h}, h=$ $1, \ldots, A_{i}$, a-priori defined. With this choice, a transition $t_{j}$ may fire, with respect to any colour, if and only if the marking after the firing is such that $\mathcal{M}_{i}^{e q} \leq C_{i}$, $\forall p_{i} \in P$.

\section{The model of urban traffic networks}

\subsection{The general framework}

From a general point of view, an Urban Transportation Network with Vehicles $(U T N V)$ can be defined as the triple

$$
\mathrm{UTNV}=\{\mathbf{V}, \mathbf{R}, \mathbf{I}\}
$$

where $\mathbf{V}=\left\{V_{l}, l=1, \ldots, \mathrm{L}\right\}$ is the set gathering the different kinds of vehicles travelling in the net, $\mathbf{R}=\left\{R_{k}, k=1, \ldots, \mathrm{K}\right\}$ is the set of the road stretches in the urban area, and $\mathbf{I}=\left\{I_{h}, h=1, \ldots, \mathrm{H}\right\}$ is the set of the intersections and the roundabouts in the urban network. In this formalisation, the set $\mathbf{V}$, which makes the present model slightly different from the one proposed by the authors in [4], on one hand requires a more powerful modelling tool with respect to the Petri Net formalism used in such a paper but, on the other hand, allows to cope the problem of modelling separately all the different kinds of vehicles moving around the seaport.

\subsection{The model of vehicles}

In the proposed model, the different kinds of vehicles of set $\mathbf{V}$ are modelled with tokens of different colours in the CSTPN. Then, the set of all the possible vehicles/colours is

$$
\mathbf{V} \equiv C l=\{\text { motorcycle, car, bus, truck }\}
$$




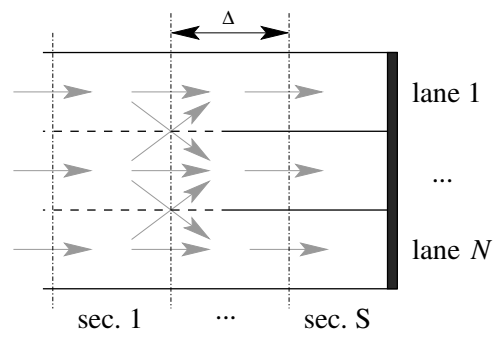

Figure 1: A $N$-lane $S$-section road stretch.

In a simplified framework, the above vehicles/colours differ among them essentially for the space that they occupy in the sections and for the turning movement, which can be allowed only for some kinds of vehicles. Note that this last characteristic is quite relevant in urban transportation networks as, for instance, when specialised lanes exist .

Then, with regard to the sizes of the different vehicles, it is possible to state that each car is equivalent to $\alpha_{c}$ motorcycles, and that each bus or truck is equivalent to $\alpha_{b}$ or $\alpha_{t}$ motorcycles, respectively. Such an approximation allows to simplify the model, since the finite space of each section of the UTN can be thought in terms of equivalent number of motorcycles. In this sense, the real space occupied by the vehicles in a section $s$, expressed in equivalent motorcycles, is

$$
n_{e q}^{s}=n_{m}^{s}+\alpha_{c} n_{c}^{s}+\alpha_{b} n_{b}^{s}+\alpha_{t} n_{t}^{s},
$$

being $n_{m}^{s}, n_{c}^{s}, n_{b}^{s}$, and $n_{t}^{s}$ the numbers of motorcycles, cars, buses, and trucks, present at the same time in section $s$, respectively. Note that such an assumption allows to introduce the capacity $C^{s}$ of sections $s$, defined in an equivalent way of the capacity of the places of the CSTPN above described.

In the following, from the CSTPN point of view, the different kinds of vehicles are graphically represented by means of tokens of different colours: black tokens represent truck, dark grey tokens represent buses, light grey tokens represent cars, and, finally, white tokens represent motorcycles.

\subsection{The model of road stretches}

Consider the $N$-lane road stretch depicted in Fig. 1. In such a figure, it is possible to note that each lane of the road stretch is divided into $S$ sections of length $\Delta$, being $\Delta$ the space occupied by a truck or a bus at a time. In addition, in the considered model, the vehicles travelling on the considered road stretch are allowed to change lane only when there is a dashed line between the lanes, as indicated by the grey arrows in Fig. 1.

In the following, for the sake of clearness, the notation $(n, s)$ will be used to indicate the $s^{\text {th }}$ section of the $n^{\text {th }}$ lane. 


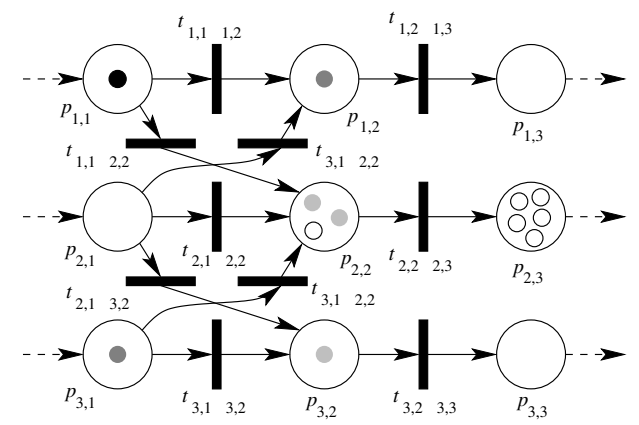

Figure 2: CSTPN model of a 3-lane 3-section road stretch.

As for the CSTPN model of the road stretch of Fig. 1, consider the net depicted in Fig. 2, which, for the sake of simplicity, is limited to a 3-lane and 3-section road model. In such a net, each place $p_{i, j}$ corresponds to a sections $(i, j)$ of the road stretch, whereas each transition $t_{i, j->h, l}$ represents the movement of vehicles from section $(i, j)$ to section $(h, l), i, j, h, l \in\{1,2,3\}$. In particular, when a transition enabled with respect to a particular colour fires, it represents the movement of the relevant kind of vehicles from a section to another one.

For instance, a car in section $(1,1)$ is represented by a light grey token (that is, a "car" token) in $p_{1,1}$. Such a token enables both the transitions $t_{1,1->1,2}$ and $t_{1,1->2,2}$, obviously with respect to the same colour "car". Then, if $t_{1,1->1,2}$ fires, the car moves to section $(1,2)$. Otherwise, if $t_{1,1->2,2}$ fires, the car moves towards section (2,2). Note that, being the timings of transitions $t_{i, j->h, l}, i, j, h, l$ $\in\{1,2,3\}$, a stochastic values, there are no conflicts between the enabled transitions, since the next transition to fire is the one with the shortest $F D$.

\subsection{The model of roundabouts}

Consider the roundabout depicted in Fig. 3. For the aim of fixing the ideas, the considered roundabout has 8 sections, 2 lanes, and 3 entries/exits. Moreover, for the same purpose, only single lanes entries/exits are considered. Finally, in the considered roundabout, all the sections are supposed to have the same (angular) dimensions $\varphi$. Then, for roundabouts with a few lanes and a big ray, all the sections result to have practically the same capacity.

In such a figure, the admissible movements of vehicles among the sections of the roundabout are also indicated. Note that starting from a section, only two other sections or, in some cases, an exit section are reachable. Moreover, from an entry only one section can be reached.

For far as it regards the CSTPN model of the roundabout of Fig. 3, consider the network depicted in Fig. 4. In the proposed CSTPN, each place represents a section and each transition represents a movement of a vehicle. In particular, each place $p_{s, n}, s=1, \ldots, 8, n=1,2$, represents the physical space of section 


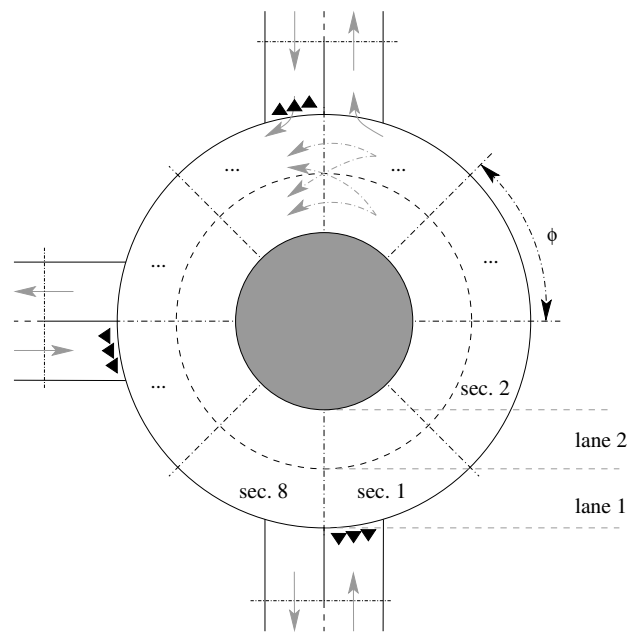

Figure 3: A 8-section 3-entrance roundabout.

$(s, n)$, whereas each place $p_{l}, l \in\{1,4,5, \ldots, 7,8\}$, represents the final section of the road stretches adjacent to the roundabout. Then, each transition $t_{s, n->k, h}$, $s, k=1, \ldots, 8, n, h=1,2$, represents the movement from section $(s, n)$ towards section $(k, h)$, if reachable. Analogously, each transition $t_{s, n->i}$ (resp., $t_{i->s, n}$ ), $s=1, \ldots, 8, n=1,2, i \in\{1,4,5, \ldots, 8\}$, represents the movement from section $(s, n)$ towards (resp., from) the road stretch sections $i$, if they are linked. Note that, due to the lack of space and to keep the model clear, only some transitions have been labelled.

To conclude, it is of paramount importance to note, in Fig. 4, the modular structure of the net. To point out such a characteristic, note that the CSTPN in the dashed box " $\mathrm{A}$ " represents a single section of a lane $(s, n)$ gathering the place $p_{s, n}$, which represents the space relevant to the section, and transitions $t_{s, n->k, h}$, which represents the movement from the considered section towards the sections reachable from $(s, n)$. On the other hand, the CSTPN in the dashed box "B" represents the last section of the input and output road stretches of the roundabout. Due to the modular structure of the net, the CSTPN "B" may be substituted by the CSTPN representing the road stretches described in Section 3.3. It is quite easy to observe that the whole CSTPN of Fig. 4, representing the roundabout of Fig. 3, is entirely built by linking several modules "A" and "B".

\subsection{The model of intersections}

As said above, the set I of an UTNV gathers not only the roundabouts above described, but also the signalised and non-signalised intersections of the net. Since, on one hand, there are no intersections in the case study described in the following Section. 4 , and, on the other hand, there is a significant amount of literature about 


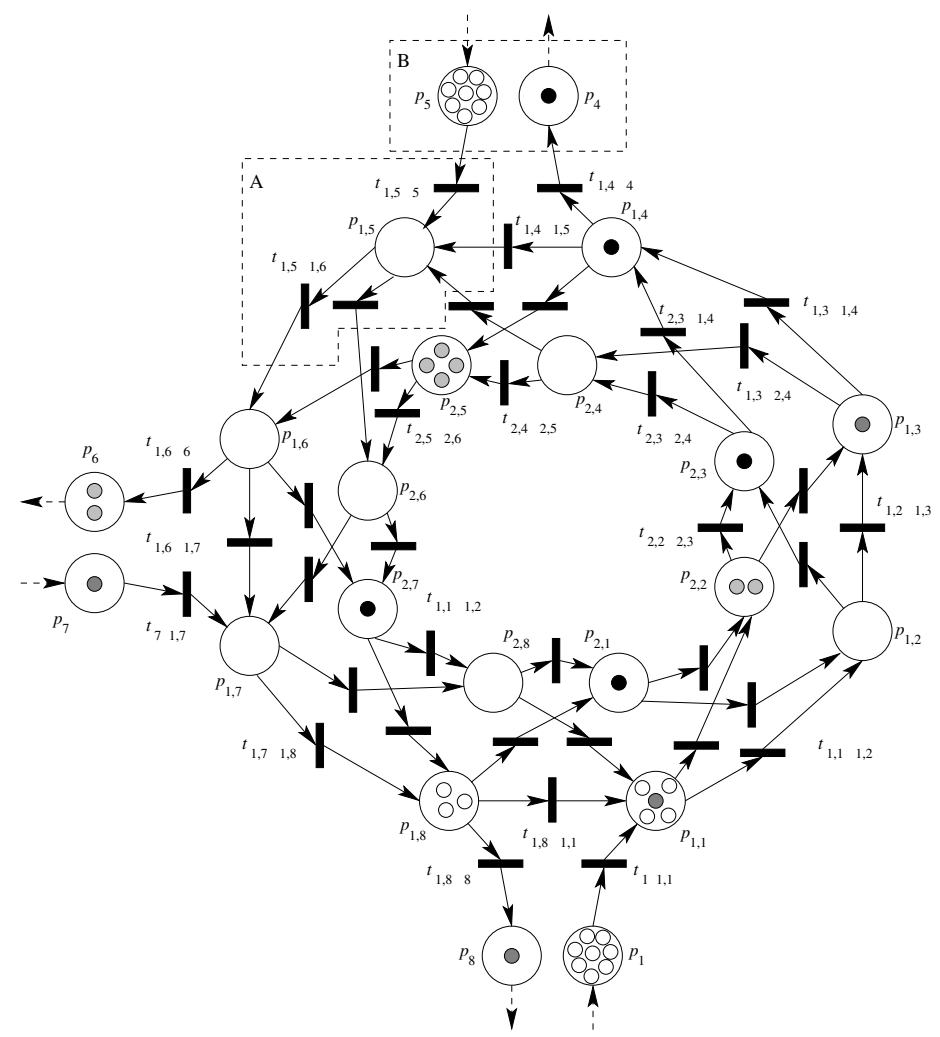

Figure 4: CSTPN model of a $S$-section 3-entrance roundabout.

them, in this paper the relevant CSTPN model is not described. The readers can refer, for instance, to [5] for a STPN model of signalised intersections, or to [1] for a valuable CSTPN model.

\section{Case study: the seaport of Genoa}

In this section, a case study is presented with the aim of pointing out the capability of the proposed modelling approach. Such a case is relevant to the seaport located in the centre of Genoa, Italy, which is the largest in the Mediterranean sea, and thus "provokes" a huge amount of trucks traffic. In effects, such an area, depicted in Fig. 5, results to be a very interesting case, study since, together with the trucks, a large number of motorcycles (which is the most common vehicle in the urban area of Genoa), cars and buses can be found there.

From a modelling point of view, in Fig. 5 it is easy to note that the whole considered network is modularly composed by several multi-lane roads, several portions of roundabout (indicated in the dashed boxes), and no intersections. The whole CSTPN model of such a network can be easily built by linking the basilar model 


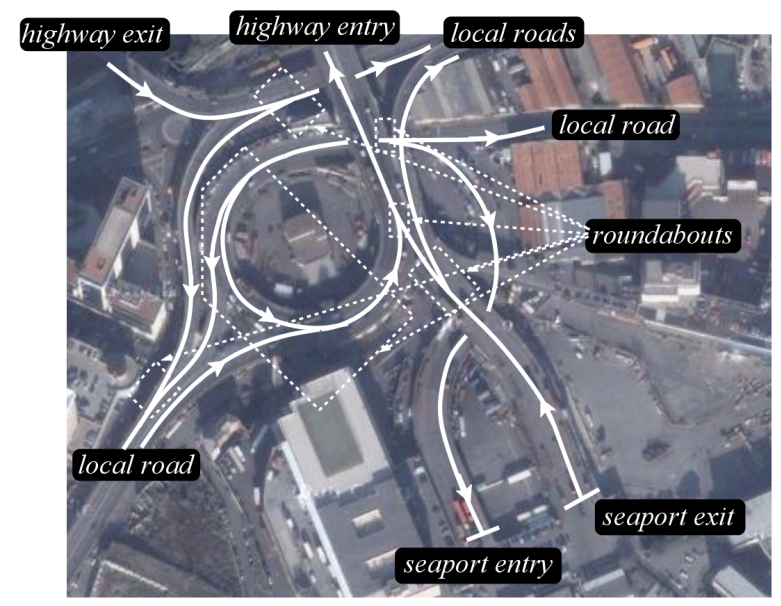

Figure 5: Road network around the gates of the seaport of Genoa.

of road stretches and roundabouts described above, and results to be a very large Petri net. Due to the lack of space, such a network is not reported here, while it is implemented into an ad-hoc $\mathrm{C}++$ simulator for further research.

\section{Conclusions}

In this paper, a CSTPN model of urban traffic network able of capturing the behaviour of different kinds of vehicles has been presented. In this framework, the main result of the paper is a model capable of modelling the different vehicles in the traffic flows, and sufficiently easy to use for building large networks.

\section{References}

[1] Dotoli, M. \& Fanti, M., An urban traffic network model via coloured timed Petri nets. Control Engineering Practice, 14(10), pp. 1213-1229, 2006.

[2] Murata, T., Petri Nets: Properties, Analysis and Applications. Proceedings IEEE, 77(4), pp. 541-580, 1989.

[3] Jensen, K., Colored Petri nets: Basic concepts, analysis methods and practical use, volume 1. Springer: New York, 1992.

[4] Di Febbraro, A. \& Sacco, N., On modelling urban transportation networks via hybrid Petri nets. Control Engineering Practice, Special issue: Analysis and Design of Hybrid Systems, 12(10), pp. 1225-1239, 2004.

[5] Di Febbraro, A., Giglio, D. \& Sacco, N., Modular Representation of Urban Traffic Systems based on Hybrid Petri Nets. Proc 2001 IEEE Int Conf on Intelligent Transportation Systems, pp. 866-871, 2001. 